\title{
The Characteristics of Stress Relaxation Curves and the Thermally Activated Deformation in Molybdenum*
}

\author{
By Koji Tanoue** and Hidehiko Matsuda**
}

\begin{abstract}
Any stress relaxation curve for polycrystalline molybdenum, obtained from stress relaxation tests by a monotonic loading mode and plotted as $\log \sigma$ vs $\log \dot{\varepsilon}$, can be superposed on each other by scaling and forms a unique master curve. The effects of such various factors as grain size (20$1500 \mu \mathrm{m})$, imposed strain rate $\left(10^{-5}-3 \times 10^{-4} \mathrm{~s}^{-1}\right)$, impurities (commercial pure molybdenum and Mo-Co alloy) and temperature $(292-550 \mathrm{~K})$ on the shape of the master curve are investigated. A deeper understanding of the characteristics and the physical meaning of the master curve have been acquired by investigating experimental results.

The primary shapes of master curves are not affected by the factors of grain size and imposed strain rate, and the master curves can also be superposed on each other by scaling. Subsequently, the stress-strain rate scaling parameter $\bar{m}\left[=(\delta \ln \dot{\varepsilon} / \delta \ln \sigma)_{v}\right]$ obtained as a reciprocal of the slope on scaling of master curves coincides with $m$ of forming each master curve. On the other hand, the master curves obtained on materials of different purities or at different temperatures cannot be superposed on each other because of their different shapes. It is concluded from the results that the different shapes of master curves may be attributed to the change in short range obstacle profiles for dislocation movement due to impurities and to the change in the effective stress due to temperature.
\end{abstract}

(Received July 11, 1983)

\begin{abstract}
Keyzerds: mechanical equation, stress relaxation, thermally activated deformation, molybdenum
\end{abstract}

\section{Introduction}

It has been demonstrated that a family of $\log \dot{\varepsilon}$ vs $\log \sigma$ curves for polycrystalline materials, obtained from stress relaxation tests after monotonic loading, can be superposed on each other by scaling and forms a unique master curve. Recent more detailed studies proved this conception to be valid for sintered pure molybdenum and its alloy, giving a mechanical equation of state as follows ${ }^{(1)(2)}$.

$$
f\left(\dot{\varepsilon}, \sigma,\left(\dot{\varepsilon}_{0}, \sigma_{i}^{0}\right), T\right)=0,
$$

where $\dot{\varepsilon}_{0}$ is a structure factor containing the mobile dislocation density, an attempt frequency and geometrical terms, and $\sigma_{i}^{0}$ the maximum internal stress at which the deformation stagnates. The scaling relation could then be described by the relation between $\dot{\varepsilon}_{0}$ (or the

* This paper was originally published in Japanese in J. Japan Inst. Metals, 46 (1982), 22.

** Department of Metal Processing Engineering, Kyushu Institute of Technology, Kitakyushu 804, Japan. mobile dislocation density) and $\sigma_{i}^{0}$ (or the total dislocation density) ${ }^{(2)}$.

The master curve, which makes it clear for the mechanical equation like the above equation to hold, gives the relation between $\dot{\varepsilon}$ and $\sigma$ on the condition that $\dot{\varepsilon}_{0}$ and $\sigma_{i}^{0}$ in eq. (1) viz. the internal structure remains almost constant. The relation can, therefore, be regarded as the locus which corresponds to a continuous drop in plastic strain rate $\dot{\varepsilon}$ and effective stress $\sigma^{*}$ until the deformation stagnates because of the thermally activated overcoming of the short range obstacles by dislocations. It follows that the master curve gives the characteristic relation between $\dot{\varepsilon}$ and $\sigma^{*}$ of the thermally activated deformation during stress relaxation and has importance to the study on rate processes in plastic deformation. However, there has been only a little amount of information about the primary nature of the master curve. It is the purpose of the present paper to clarify the characteristics and the physical meaning of master curves by investigating the effects of such various factors as grain size, imposed strain rate, impurities and temperature on their shapes. 


\section{Experimental Procedure}

Materials used in the present work are powder-metallurgically pure molybdenum wires $(\phi 1.3 \mathrm{~mm})$ and plates $(1 \mathrm{~mm}$ thickness) of commercial grade (above $99.95 \%$ ) and wires $(\phi 1.3 \mathrm{~mm})$ of $\mathrm{Mo}-0.05 \mathrm{at} \% \mathrm{Co}$ alloy. Table 1 shows the chemical composition of the materials. The wires and the plates were electropolished, after they were mechanically shaped to be $\phi 1.2 \mathrm{~mm} \times 50 \mathrm{~mm}$ and $1 \mathrm{~mm} \times 3 \mathrm{~mm} \times$ $50 \mathrm{~mm}$, respectively. The specimens were then annealed in a vacuum of $2 \times 10^{-4} \mathrm{~Pa}$ to obtain various grain sizes. The resultant mean grain sizes were 17 to $32 \mu \mathrm{m}$ for pure Mo wires and $23 \mu \mathrm{m}$ for Mo-Co alloy wires. The four nominal grain sizes of $30,50,100$ and $1500 \mu \mathrm{m}$ were prepared for the plates by a double strain annealing procedure, the details of which were reported elsewhere ${ }^{(3)}$.

Simple tension and stress relaxation tests were made using an Instron type tensile testing machine, Shimadzu IS-500. The specimens with the gage length of $25 \mathrm{~mm}$ were deformed at the nominal strain rate of $\dot{\varepsilon}=3.3 \times 10^{-4} \mathrm{~s}^{-1}$ and the five test temperatures of 292 (300 on the plates), 350,420 (400 and 450 on the plates), 500 and $550 \mathrm{~K}$. The imposed strain rates $\dot{\varepsilon}$ were changed variously to be $10^{-5}, 10^{-4}$ and $3.3 \times$ $10^{-4} \mathrm{~s}^{-1}$, in order to investigate their effect on the shape of master curves. Further details of the procedure to obtain a family of $\log \dot{\varepsilon}$ vs $\log \sigma$ curves have been presented in the previous paper $^{(2)}$. All of the present master curves were constructed by translation of the stress relaxation curves after monotonic loading onto the curve with about $1 \%$ strain.

\section{Experimental Results}

Figure 1 shows the stress relaxation curves for pure Mo wires at $292 \mathrm{~K}$. The plastic strain rates at the beginning of relaxation agree with the imposed strain rate. These curves can be superposed on each other by translation along the oblique line and form a master curve. The scaling parameter $m$ was obtained to be 8.6 as a reciprocal of the slope on scaling according to the following definition.

$$
m=1 / \mu=(\delta \ln \dot{\varepsilon} / \delta \ln \sigma)_{v},
$$

where $v$ is a tangent on the relaxation curves and $\delta$ signifies the small change in $\ln \sigma$ and $\ln \dot{\varepsilon}$ on the scaling line. Figure 2 represents the master curves for pure Mo wires with several

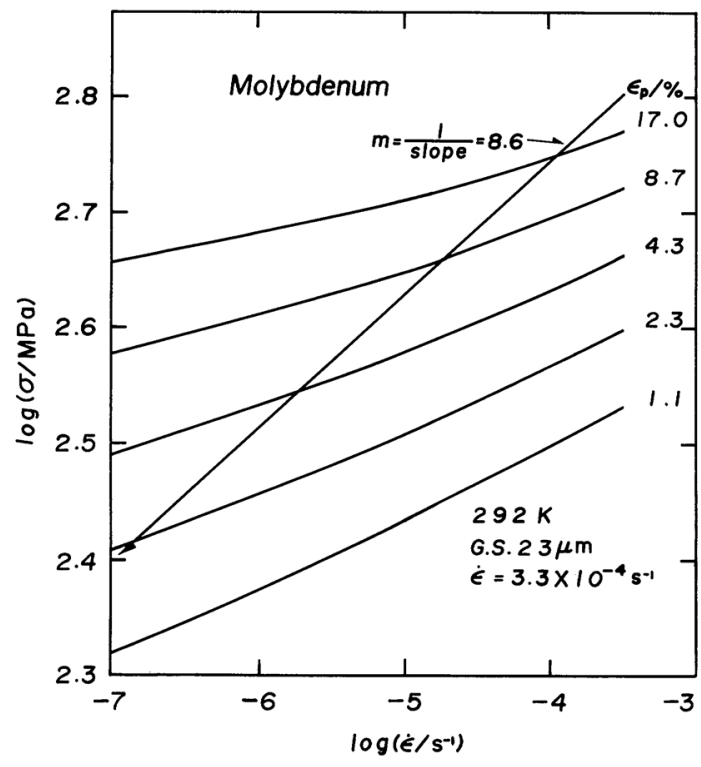

Fig. 1 A series of stress relaxation curves following monotonic loading for pure molybdenum wires at $292 \mathrm{~K}$. The straight line shown represents the scaling relation.

Table 1 Chemical composition of specimens.

(ppm in mass)

\begin{tabular}{lcrrrrrrr}
\hline \hline \multicolumn{1}{c}{ Materials } & $\mathrm{Fe}$ & $\mathrm{Ni}$ & $\mathrm{Cu}$ & $\mathrm{Co}$ & $\mathrm{O}$ & $\mathrm{N}$ & $\mathrm{C}$ & NVR$^{*}$ \\
\hline Pure Mo (wire) & 20 & 10 & $<30$ & - & 26 & 8 & $<30$ & 10 \\
Pure Mo (plate) & 80 & $<30$ & $<30$ & - & 160 & 7 & $<30$ & 40 \\
Mo-Co alloy & 20 & 10 & - & 510 & - & - & - & 10 \\
\hline \hline
\end{tabular}

* Non volatilized residuum containing $\mathrm{Al}, \mathrm{Ca}, \mathrm{Si}$ and $\mathrm{Mg}$. 
grain sizes at $292 \mathrm{~K}$. Each master curve, constructed by translation of the original relaxation runs with the slope of $1 / 8.6$, could again be superposed with the same slope. Therefore, $m$ agreed with $\bar{m}$ for the master curves.

The master curves obtained by changing the imposed strain rates variously are presented in

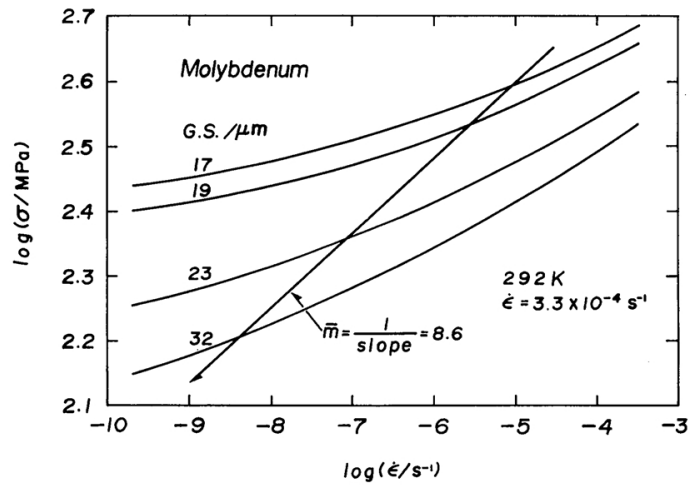

Fig. 2 Master curves for pure molybdenum wires with several grain sizes at $292 \mathrm{~K}$. Each master curve is constructed by superposing a series of stress relaxation curves onto the curve with about $1 \%$ plastic strain.

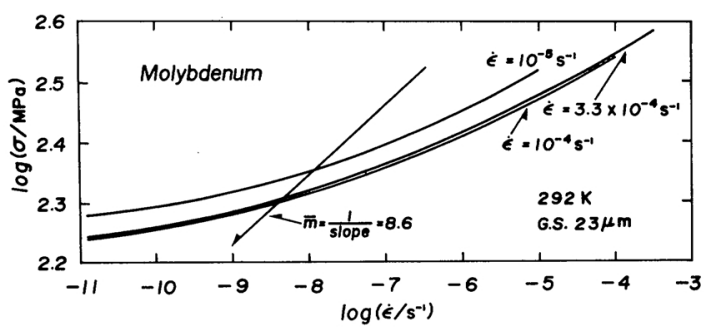

Fig. 3 Master curves with various imposed strain rates on monotonic loading for pure molybdenum wires at $292 \mathrm{~K}$.

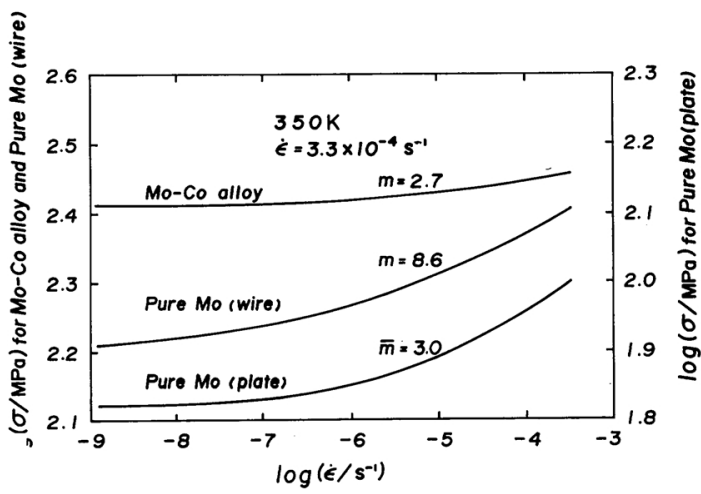

Fig. 4 Master curves for several materials at $350 \mathrm{~K}$.
Fig. 3, taking pure Mo wires with $23 \mu \mathrm{m}$ grain size at $292 \mathrm{~K}$ as an example. It is seen from the figure that the initial plastic strain rates of relaxation agree with the imposed strain rates exactly, and that the curves can be scaled with the same $m$ value as in Fig. 2. Figure 4 shows the master curves for several materials at $350 \mathrm{~K}$. The curves for the Mo-Co alloy and pure Mo wires were obtained using the specimens with $23 \mu \mathrm{m}$ grain size, whereas another curve for the plates was constructed from the additional translation of four master curves with the nominal grain sizes of 30,50, 100 and $1500 \mu \mathrm{m}$. On the latter scaling, $m$ and $\bar{m}$ were, therefore, obtained for each master curve and all of them, respectively, leading to $m=\bar{m}=3.0$. No scaling relation held among the curves, because $m$ and their curvature were different from each other.

The master curves for pure Mo wires are rewritten as $\sigma$ vs $\log \dot{\varepsilon}$ and shown in Fig. 5 . These curves were obtained using the specimens with $23 \mu \mathrm{m}$ grain size except one at $292 \mathrm{~K}$, which was constructed by superposing master curves in Fig. 2 onto the curve with $32 \mu \mathrm{m}$ grain size, yielding $\sigma_{i}^{0}=110 \mathrm{MPa}$. All of the curves are illustrated to discuss only about the effective stress $\sigma^{*}\left(=\sigma-\sigma_{i}^{0}\right)$ after translating the flow stress along the ordinate so that $\sigma_{i}^{0}$ may coincide with each other ${ }^{\dagger}$. The plastic strain

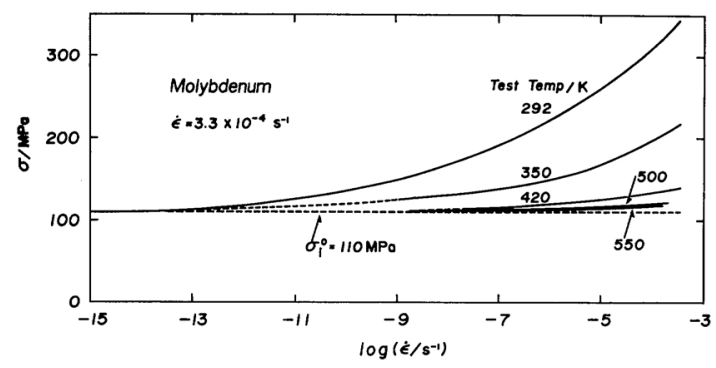

Fig. 5 The relation of $\sigma$ versus $\log \dot{\varepsilon}$ for pure molybdenum wires as a function of temperature.

$\dagger$ Though three curves for 292, 500 and $550 \mathrm{~K}$ covered the entire range to deformation stagnation, the other curves were insufficient. Therefore, the initial flow stresses $\sigma_{0}$ of master curves at 350 and $420 \mathrm{~K}$ for $\sigma_{\mathrm{i}}^{0}=110 \mathrm{MPa}$ were estimated from $\sigma_{0}=$ $\sigma_{i}^{0}+\sigma_{i}^{*}$. Here, the effective stress $\sigma_{1}^{*}$ was calculated from $\sigma_{1}^{*}=2 k T m^{*} / V^{*}$ with the dislocation velocitystress exponent $m^{*}$ and the activation volume $V^{*}$, which were obtained using the same material at $\dot{\varepsilon}=3.3 \times 10^{-4} \mathrm{~s}^{-1}$ as reported in ref. (4). 
rates at the beginning of relaxation did not necessarily coincide with the imposed strain rates at 500 and $550 \mathrm{~K}$. This means that $\sigma^{*}$ is very small at the temperatures ${ }^{(1)(4)}$. Because $m$ for each master curve decreased drastically with increasing temperature, these master curves could not be scaled.

\section{Discussion}

\section{Effect of various factors on the shape and the scaling locus of master curves}

The scaling parameter $m$ varied with the amount of impurities and temperature, whereas $m$ remained constant independent of grain size and imposed strain rate. It is interpreted that the former factors cause $m$ to vary by affecting the relation between $\dot{\varepsilon}_{0}$ (or $\rho_{m}$ ) and $\sigma_{i}^{0}$ as reported in the previous paper ${ }^{(2)}$. Figures 2 and 3 show that the latter factors have no effect on the primary shape of master curves and the scaling is possible, and that their different shapes are due to the different internal stresses $\sigma_{i}$ (or $\sigma_{i}^{0}$ ). The agreement between $m$ and $\bar{m}$ also implies that these factors produces no significant change in the relation between $\dot{\varepsilon}_{0}$ and $\sigma_{i}^{0}$. If both $m$ and the shape of master curves vary simultaneously, the scaling among them becomes impossible, as seen in Fig. 4. The different shapes of master curves like this figure may be attributed to the change in short-range obstacle profiles for dislocation movement as described later. However, the different shapes such as Fig. 5 do not result from the different short-range obstacle profiles but from the change in $\sigma^{*}$ due to temperature, because these curves construct a single profile as shown in Fig. 9. It should also be noted that though $\dot{\varepsilon}$ changes the starting point of the relaxation run by affecting $\sigma^{*}$ as seen in Fig. 3, it has no effect on the shape of master curves in contrast with the results in Fig. 5.

\section{The characteristics of master curves based on the thermally activated deformation}

The effective stress $\sigma^{*}$ relating only to the thermally activated deformation is defined by $\sigma^{*}=\sigma-\sigma_{i}^{0}$, and the logarithmic plot of $\sigma^{*}$

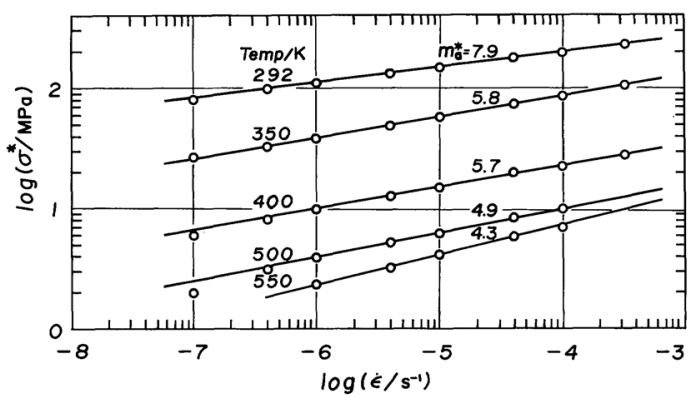

Fig. 6 Logarithmic plot of the effective stress $\sigma^{*}$ against the plastic strain rate $\dot{\varepsilon}$ at various temperatures for pure molybdenum wires.

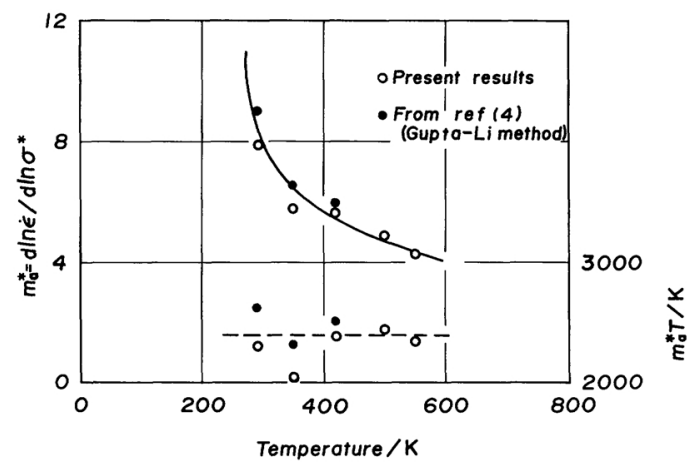

Fig. 7 Variation of the apparent dislocation velocitystress exponent $m_{a}^{*}$ with temperature for pure molybdenum wires.

against $\dot{\varepsilon}$ is given in Fig. 6. The experimental data are well ruled by a linear relationship at any temperature. The apparent dislocation velocity-stress exponent $m_{a}^{*}\left(=\mathrm{d} \ln \dot{\varepsilon} / \mathrm{d} \ln \sigma^{*}\right)$ is obtained from the slope and shown in Fig. 7. In the figure, results ${ }^{(4)}$ obtained from the Gupta-Li method ${ }^{(5)}$ are also plotted, which coincide the present results approximately. As the master curves are obtained on the condition that $\dot{\varepsilon}_{0}$ (or $\rho_{m}$ ) is constant, the change in $\dot{\varepsilon}$ $\left(\propto \rho_{m} \bar{v}\right)$ during stress relaxation corresponds to the change in the mean dislocation velocity $\bar{v}$. Accordingly, $m_{a}^{*}$ can be regarded as the true dislocation velocity-effective stress exponent $m^{*}\left(=\mathrm{d} \ln \bar{v} / \mathrm{d} \ln \sigma^{*}\right)$. It is found from the above results that the relation between $\dot{\varepsilon}$ and $\sigma^{*}$ can be described by the Johnston-Gilman ${ }^{(6)}$ type equation as follows.

$$
\dot{\varepsilon}=\alpha \rho_{m} b \bar{v}=\alpha^{\prime} \rho_{m} b \sigma^{* m^{*}},
$$

where $\alpha$ is a geometrical factor and $\alpha^{\prime}$ a constant containing $\alpha$. 
On the other hand, the plastic strain rate $\dot{\varepsilon}$ during stress relaxation can also be expressed by

$$
\dot{\varepsilon}=\dot{\varepsilon}_{0} \exp \left[-\Delta G\left(\sigma^{*}\right) / k T\right]
$$

where $T$ is the temperature, $k$ the Boltzmann constant and $\Delta G$ the Gibbs free energy which is a decreasing function of effective stress. If $\dot{\varepsilon}_{0}$ does not depend on temperature, one can obtain $\Delta G$ from the Arrhenius plot of $\ln \dot{\varepsilon}$ against $1 / T$. The plot at each level of effective stress is presented in Fig. 8. The linear relationship holds, and $\Delta G$ decreases as $\sigma^{*}$ increases, giving $\Delta G$ to be $0.20 \mathrm{aJ}$ at $\sigma^{*}=10 \mathrm{MPa}$. This value agrees with the activation energy (or enthalpy) $\Delta H_{0}=0.21 \mathrm{aJ}^{(7)}$ at $500 \mathrm{~K}$ estimated from the linear relationship of $\Delta H$ against $T$ where $\sigma^{*}$ becomes almost zero at this temperature.

Thus, the dependence of $\dot{\varepsilon}$ on $\sigma^{*}$ and $T$ satisfied well eqs. (3) and (4), respectively. If the dependence of $\dot{\varepsilon}$ on $\sigma^{*}$ is also expressed by eq. (4), both equations become equal in the dependence of $\dot{\varepsilon}$ on $\sigma^{*}$, only when $\Delta G\left(\sigma^{*}\right)$ is given by

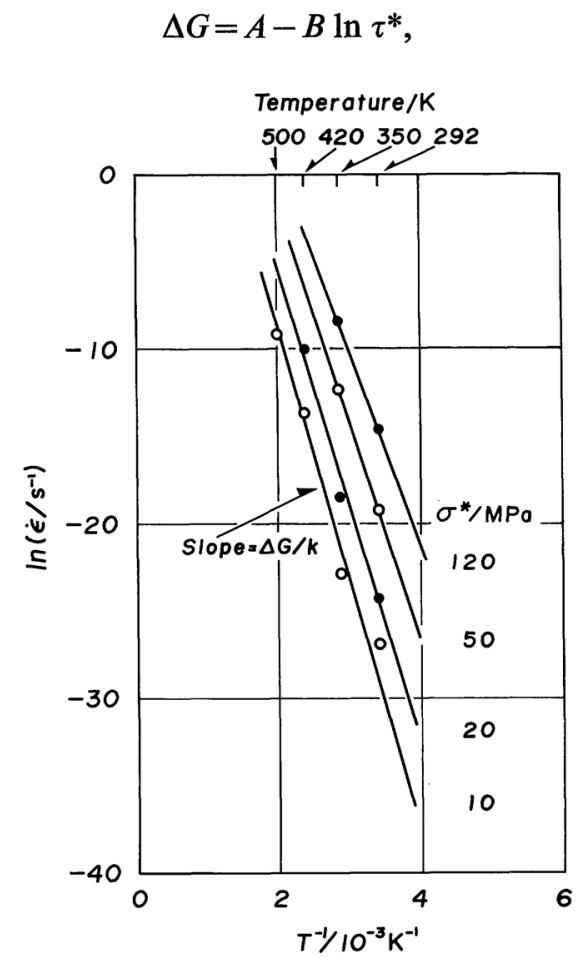

Fig. 8 The Arrhenius plot of the plastic strain rate against the reciprocal of temperature for pure molybdenum wires. where $A$ and $B$ are constants and $\tau^{*}\left(=1 / 2 \cdot \sigma^{*}\right)$ the effective shear stress. If the low temperature deformation ( $T<0.25 T_{m}, T_{m}$ the melting point) in molybdenum is controlled by a single mechanism of the thermally activated overcoming of the Peierls-Nabarro hills by the double kink nucleation, the activation energy $\Delta H$ for the process at low stresses is given by the following equation $^{(8)}$.

$$
\Delta H=H_{k}\left(1+\frac{1}{4} \ln \frac{16 \tau_{p}}{\pi \tau^{*}}\right),
$$

where $H_{k}$ is the kink energy and $\tau_{p}$ the Peierls stress. From $\Delta G=\Delta H-T \Delta S$, eq. (6) turns out to be an example of eq. (5). Combination of eqs. (4), (6) and $\Delta G=\Delta H-T \Delta S$ gives

$$
\dot{\varepsilon}=\dot{\varepsilon}_{0} \exp (\Delta S / k) \cdot\left(\tau^{*} / D\right)^{H / 4 k T},
$$

where $\Delta S$ is the activation entropy and $D$ a constant equal to exp $\left[4+\ln \left(16 \tau_{p} / \pi\right)\right]$. Regarding $H_{k} / 4 k T$ as $m^{*}$, eq. (7) agrees with eq. (3) in the dependence of $\dot{\varepsilon}$ on $\sigma^{*}$ and accounts for the results in Figs. 6 and 8. If $H_{k}$ does not depend on temperature, $m^{*} T\left(=H_{k} / 4 k\right)$ becomes constant. The measured values of $m_{a}^{*} T$ are shown in Fig. 7, being approximately independent of temperature. The kink energy $H_{k}$ $\left(=4 \mathrm{~km}^{*} T\right)$ was then calculated to be $0.13 \mathrm{aJ}$ which agreed well with 0.11 aJ reported by Prekel and Conrad ${ }^{(9)}$ using the etch-pitting technique for high purity molybdenum single crystals.

Another expression for the dependence of $\Delta G$ on $\tau^{*}$ can be given by the following phenomenologically derived equation ${ }^{(10)}$.

$$
\Delta G=\Delta G_{0}\left[1-\left(\tau^{*} / \tau_{p}\right)^{p}\right]^{q},
$$

where $\Delta G_{0}$ is the Gibbs free energy when $\tau^{*}$ is zero, and $p$ and $q$ the parameters dependent on the profile of short range obstacles for dislocation movement. All published models ${ }^{(11)-(13)}$ for the Peierls mechanism can almost be expressed by eq. ( 8 ) with $p=3 / 4$ and $q=4 / 3$ except for eq. (6) by Seeger ${ }^{(8)}$. The activation volume $V^{*}$ was calculated from eq. (8) according to the definition of $V^{*}=-\partial \Delta G / \partial \tau^{*}$ and shown in Fig. 9 as the relation of $\tau^{*} / \tau_{p}$ versus $V^{*} \tau_{p} / \Delta G_{0}$. Here, the calculation was made using $569 \mathrm{MPa}^{(4)}$ as $\tau_{p}$ and $0.20 \mathrm{aJ}$ at $\sigma^{*}=10 \mathrm{MPa}$ in Fig. 8 as $\Delta G_{0}$. The two dotted lines with $p=1$, 


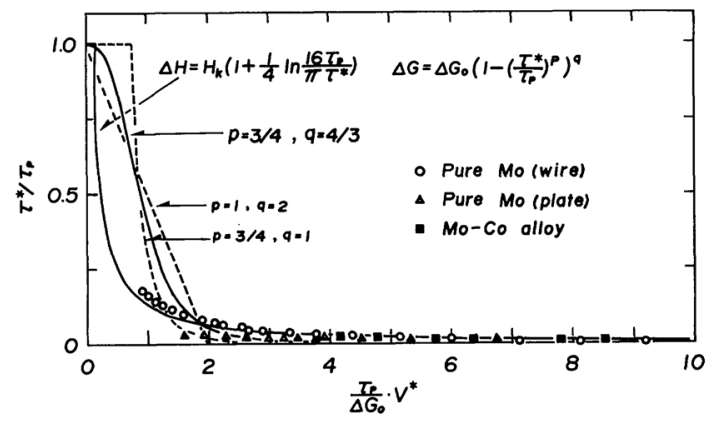

Fig. 9 Short-range obstacle profiles described by the relation of $\tau^{*} / \tau_{p}$ versus $V^{*} \tau_{p} / \Delta G_{0}$ for several materials.

$q=2$ and $p=3 / 4, q=1$ denote the profiles deviated from the average form $(p=3 / 4, q=$ $4 / 3$ ) of the activation energy of double kink nucleation. While the former is obtained on the assumption that the shape of the Peierls potential is quasi-parabolic, the latter is deduced from the model with a pair of separated kinks $^{(10)}$. By getting $(\mathrm{d} \sigma / \mathrm{d} \ln \dot{\varepsilon})_{T}$ from Fig. 5, the activation volume $V^{*}\left[=2 k T(\mathrm{~d} \ln \dot{\varepsilon} / \mathrm{d} \sigma)_{T}\right]$ is calculated and shown in Fig. 9 as a function of $\tau^{*}$. Any data points produced one smooth array in spite of different temperatures, falling on the lower stress level with increasing temperature. The figure also contains the results for pure Mo plates and the Mo-Co alloy. The data points for pure Mo wires satisfies the relation of $V^{*} \tau_{p} / 2 H_{k}=\tau_{p} / 8 \tau^{*}$ deduced from eq. (6) rather than the average form with $p=3 / 4$ and $q=4 / 3$. Conversely, the data points for the plates were situated on the other profile apart from the Mo-Co alloys of which positions are obscure because of very small $\tau^{*} / \tau_{p}$. It seems, therefore, that the invalidity of the mutual scaling among the master curves for several materials, of which curvatures and $m$ were different from each other as seen in Fig. 4, results from the change in short-range obstacle profiles for dislocation movement.

In low concentration alloys, the dislocation velocity is controlled by the rate of formation of double kinks at low temperatures. As the activation energy of the double kink formation varies with the fluctuation in the number of solute atoms at the site of double kink formation, a double kink may preferentially be formed at the site where the activation energy decreases.
This reasoning accounts for the solution softening at low temperatures ${ }^{(14)(15)}$. When the wires and the plates of pure Mo were deformed at the same strain rate, temperature $(292 \mathrm{~K})$ and grain size $(30 \mu \mathrm{m})$, the plates showed a decrease of about $70 \mathrm{MPa}$ in flow stress at each strain compared with the wires. Therefore, the impurities have a significant effect on the deformation in the present materials. The comparison of $V^{*}$ for both materials at the same level of $\tau^{*}$ shows that $V^{*}$ for the plates is lower than that for the wires, as seen in Fig. 9. It is clear from Table 1 that a fair amount of impurities in the plates affects the activation energy for the double kink formation and changes the dependence of $\Delta G$ on $\tau^{*}$, yielding the difference in $V^{*}$.

As described above, the dependence of $\dot{\varepsilon}$ on $\sigma^{*}$ and $T$ for pure Mo wires could be expressed by eq. (6) based on the Peierls mechanism. At low temperatures, the activation energy for double kink formation may depend on the material used, because it is influenced by impurities. Consequently, the change in $\Delta G\left(\tau^{*}\right)$ or the force-distance curve for short range obstacles due to impurities affects the primary shape of master curve, and the scaling among them becomes impossible. On the contrary, the scaling is possible, even if such factors as grain size and imposed strain rate are changed variously, because they do not affect the forcedistance curve. When both of the effective stress and the relation between $\dot{\varepsilon}_{0}$ and $\sigma_{i}^{0}$ (or $m$ ) change simultaneously with temperature, the scaling is no longer possible. However, this does not mean the change in $\Delta G\left(\tau^{*}\right)$ or the shortrange profiles with temperature.

\section{Conclusion}

A family of stress relaxation curves for polycrystalline molybdenum, obtained from stress relaxation tests after monotonic loading and plotted as $\log \dot{\varepsilon}$ vs $\log \sigma$, can be superposed on each other by scaling and forms a unique master curve. The characteristics and the physical meaning of master curves are clarified by investigating the effects of such various factors as grain size, imposed strain rate, impurities and temperature on their shapes. The results are summarized as follows. 
(1) The primary shapes of master curves were not affected by the factors of grain size and imposed strain rate, and the master curves could also be superposed on each other by scaling. Consequently, the scaling parameter $\bar{m}(=1 / \bar{\mu})$ obtained as a reciprocal of the slope on scaling of master curves coincided with $m$ on forming each master curve.

(2) The master curves obtained by changing the amount of impurities and temperature could not be superposed on each other because of the different shapes of the master curves. The different amount of impurities may cause the force-distance curve for short range obstacles to change by affecting the activation energy for double kink formation, and the scaling becomes impossible. On the other hand, the primary shape of master curves remains constant independent of temperature, because their different shapes can attribute only to the change in effective stress.

(3) The relation between $\dot{\varepsilon}$ and $\sigma^{*}$ obtained from the master curves for pure Mo wires satisfies well eq (6) based on the Peierls mechanism. The various activation parameters such as the dislocation velocity-effective stress exponent $m^{*}$, the kink energy $H_{k}$ and the Gibbs free energy $\Delta G_{0}$ at $\sigma^{*} \simeq 0$ were characteristic of the Peierls mechanism, showing that the ratecontrolling mechanism during stress relaxation was the thermally activated overcoming of the Peierls-Nabarro hills by the double kink nucleation.

\section{Acknowledgements}

The authors are grateful to Nippon Tungsten Co., Ltd. and Education Center for Information Processing of Kyushu Institute of Technology for their support of this research.

\section{REFERENCES}

(1) K. Tanoue and H. Matsuda: Trans. JIM, 23 (1982), 234.

(2) K. Tanoue, O. Nakano and H. Matsuda: Trans. JIM, 24 (1983), 205.

(3) K. Tanoue and H. Matsuda: Trans. JIM, 23 (1982), 727.

(4) K. Tanoue, K. Senba and K. Okazaki: J. Japan Inst. Metals, 40 (1976), 632 (in Japanese).

(5) I. Gupta and J. C. M. Li: Met. Trans., 1 (1970), 2323.

(6) W. G. Johnston and J. J. Gilman: J. Appl. Phys., 30 (1959), 129.

(7) K. Tanoue: Thesis for Doctorate of Engineering, Kyushu University, (1980), 150 (in Japanese).

(8) A. Seeger: Phil. Mag., 1 (1956), 651.

(9) H. L. Prekel and H. Conrad: Dislocation Dynamics, Ed. by A. R. Rosenfield et al., McGrawHill, (1968), p. 431.

(10) U. F. Kocks, A. S. Argon and M. F. Ashby: Progress in Materials Science, Ed. by B. Chalmers, J. W. Christian and T. B. Massalski, Pergamon Press, New York, Vol. 19 (1975), p. 110, p. 171.

(11) J. E. Dorn and S. Rajnak: Trans. Met. Soc. AIME, 230 (1964), 1052.

(12) J. Friedel: Dislocations, Addison-Wesley, Reading, Mass., (1964), p. 49.

(13) J. Lothe and J. P. Hirth: Phys. Rev., 115 (1959), 543.

(14) A. Sato and M. Meshii: Acta Met., 21 (1973), 753.

(15) H. Suzuki: Rate Processes in Plastic Deformation of Materials, Ed. by J. C. M. Li and A. K. Mukherjee, ASM, (1975), p. 47. 